\title{
BESPRECHUNGSAUFSATZE
}

\section{Wirtschaftsprobleme in Entwicklungsländern}

\author{
Von Ludwig Gramlich
}

Benno Engels (Hrsg.)

Handelsförderung für Entwicklungsländer

Deutsches Übersee-Institut, Hamburg 1989, DM 28,-

Jürgen Oestereich

Ländliche Entwicklung und Selbsthilfeförderung

Weltforum Verlag, Köln 1989, DM 25,-

Jürgen Reinhardt, Omar Shamleh, Christian Uhlig

Der Dienstleistungssektor ausgewählter Entwicklungsländer: Entwicklungs- und handelspolitische Aspekte

Weltforum Verlag, Köln 1989, DM 30,-

Die mißliche Situation vieler Staaten/Völker der "Dritten Welt" - tief verschuldet gegenüber privaten wie öffentlichen Finanzinstitutionen vorab der westlichen Industrieländer, gebunden im Hinblick auf notwendige Exportsteigerungen durch Einfuhrrestriktionen der Gläubigerstaaten, neuerdings zudem gehemmt durch globale ökologische Zwänge, die ihre bisher als natürlich erachteten (Standort-)Vorteile mehr und mehr verringem - ist Grundthema aller drei hier anzuzeigenden Arbeiten, welche sich je spezifisch mit den Möglichkeiten einer Verbesserung der Lage in Entwicklungsländern befassen.

Der von Engels edierte Sammelband mit den Beiträgen der 4. Außenwirtschaftstagung des Übersee-Instituts setzt durchweg verstärkt auf privatwirtschaftliche Aktivitäten. Nach einer Skizze der "Rahmenbedingungen und Aufgaben" durch den Herausgeber selbst widmet sich Koopmann der EG-Handelspolitik und endet mit der Befürchtung, dort werde weiterhin eine "Pyramide von Privilegien" Bestand haben (30). Khan erörtert die Bedeutung der kurz vor ihrem Abschluß befindlichen Uruguay-Runde des GATT für minder entwickelte 
Staaten; deren Halbzeitergebnis zumindest sei unbefriedigend gewesen (59), eine Reform des Abkommens im Sinne seiner Stärkung geboten (50 f.). Hoffmann bewertet positiv die Zusammenführung von potentiellen Käufern und Lieferanten durch (halb)staatliche Organisationen vor allem in den "Schwellenländern" (67 f.). Ein umfangreicherer Beitrag von Kirchbachs zeigt die Schlüsselrolle von Vermarktungskanälen bei der Ausfuhrförderung auf und plädiert für Koexistenz von öffentlichen mit privaten Unternehmen (der Entwicklungsländer) und von transnationalen Korporationen (90). Von Gleich ist um eine Systematisierung der Ziele, Wege und Instrumente von Handelsförderung bemüht; dies wäre freilich wohl besser am Anfang des Buches erfolgt. Fallstudien hinsichtlich der Exportanreize einzelner Länder bieten Machetzki (südostasiatische Region), Fasbender und Naimi mit einem überaus instruktiven Vergleich zwischen Indien und Indonesien sowie Alkazaz (arabische Staaten). Pohl erörtert das komplementäre Problem - Erhöhung der Einfuhren aus Entwicklungsländern - am Beispiel Japans. Das niederländische Centre for the Promotion of Imports from Developing Countries (CBI) ist mit einer Selbstdarstellung vertreten; dem schließt sich ein "Orientierungsrahmen" des Bundesministeriums für wirtschaftliche Zusammenarbeit zur Förderung der Privatwirtschaft in der "Dritten Welt" an, welcher eine Kooperation mit Nicht-Regierungs-Organisationen befürwortet (185). Im Anhang sind ältere, zudem undatierte Dokumente abgedruckt: Offenbar sollen sie belegen, daß "aid by trade" kein neues Konzept sei. Leider sind Qualität und wissenschaftliche Aufbereitung der diversen Beiträge überaus unterschiedlich ausgefallen.

Oestereichs Forschungsbericht für das BMZ - zusammen mit senegalesischen Autoren abgefaßt - bemüht sich, am Fall des westafrikanischen Landes, dessen Spezifik durchaus gesehen wird (24), gewonnene Erkenntnisse über "Ländliche Entwicklung und Selbsthilfeförderung" zu verallgemeinern; diesem Zweck dürfte die vorangestellte knappe Zusammenfassung dienlich sein. Oestereich erörtert zunächst "die großen Linien der spontan im Wechselspiel mit den soziopolitischen Bedingungen entstandenen sozialen Bewegung der ländlichen Selbsthilfe" (25) und betont dabei den erforderlichen Manövrierraum für (inter)nationale Nicht-Regierungs-Organisationen als Kooperationspartner (36 f.). Kritisch beurteilt wird das bisherige staatliche Verhalten den vielfältigen Selbsthilfegruppen gegenüber; Oestereich sieht allerdings gewisse Chancen für eine akzeptable Zusammenarbeit (102 f.), trotz des zentralistischen Modells von Daseins"für"sorgeaktivitäten. Staatliche Stellen müßten die geeigneten Rahmenbedingungen für (finanzielle) Autonomie, Diversifizierung (der Aktivitäten) und Konzertierung (der verschiedenen Gruppen) garantieren (121); dabei dürfe die komplexe soziale Motivation als Triebfeder von Selbsthilfe (126) nicht beschnitten werden. Abschließend beleuchtet Oestereich die insoweit erforderliche Modifikation der bilateralen Entwicklungs-Zusammenarbeit mit der Bundesrepublik Deutschland (135 ff.); diese sei vonnöten, um einen Zusammenbruch der ökonomischen Hoffnungen der Landbevölkerung (im Senegal und anderswo) zu vermeiden (141). Die Vorschläge bleiben jedoch eher vage und werden nicht weiter auf ihre Implementierbarkeit untersucht. 
Aus dem Institut für Entwicklungsforschung und Entwicklungspolitik der Universität Bochum stammt die dritte, ebenfalls für das BMZ gefertigte Studie, die der Unterschätzung des tertiären Sektors für den Entwicklungsprozeß entgegenwirken will (V), nicht zuletzt mit Blick auf die Einbeziehung des grenzüberschreitenden Dienstleistungsverkehrs in den GATT-Rahmen. So widmet sich Teil A dem Forschungsstand, um die späteren Fallstudien auf festem Grund anzusiedeln, wobei eine funktionale Einteilung gewählt wird (5 f.). Die Autoren kommen zum Fazit, differenzierte Länder-Untersuchungen seien nötig (32); die für die Detailanalyse ausgesuchten Staaten verbinde ihre Klassifikation als "middle-income countries", in geographischer Lage und handelspolitischer Ausrichtung wiesen Malaysia, Jordanien und Zimbabwe durchaus spezifische Besonderheiten auf. Die drei Länderstudien befassen sich je vorab mit wichtigen Strukturdaten und der aktuellen wirtschaftlichen Entwicklung, leisten dann eine entwicklungs- und außenwirtschaftliche Analyse des Dienstleistungsbereichs im einzelnen und stellen vor einem Resumé die nationale(n) Dienstleistungspolitik(en) dar. Dabei zeigen sich überraschend viele Ähnlichkeiten, vor allem die erhebliche Bedeutung dieses Zweiges der Volkswirtschaften für die Wertschöpfung und Beschäftigung und die dort vorhandene, beachtliche Produktivität (226 ff.). Teil C versucht schließlich, hieraus Elemente einer entwicklungsfördernden Dienstleistungspolitik in den Untersuchungsländern zu deduzieren: verstärkte Förderung des "Humankapitals" (232) nicht zuletzt angesichts der Bedeutungszunahme der Informations- und Kommunikationstechniken, aber auch mehr Bemühen im Tourismussektor wegen dessen Devisenerlöspotentialen (236f.). Allen drei Staaten wird eine engagierte Beteiligung an der Uruguay-Runde nahegelegt (239 ff.), deren Auswirkungen bislang offenbar nicht (genau) gesehen würden. Wenn die Autoren den Untersuchungsländem "prinzipiell die schrittweise, selektive Offnung des Dienstleistungssektors" (240) empfehlen, so verkennen sie nicht, daß - so der Schlußsatz - "die Liberalisierungsfrage im Falle etwa von wirtschaftlich weniger entwickelten Ländern, geschweige denn von Least Developed Countries wegen einer dort zu vermutenden schwächeren Dienstleistungsstruktur wahrscheinlich sehr viel zurückhaltender zu beantworten ist" (241). Die durchweg und zudem in einem Tabellenanhang mit reichem statistischen Material unterlegte Gemeinschaftsarbeit gewinnt ihre große Informationsvermittlungsfunktion vor allem dadurch, daß sie den Aspekt der mit Handel und sonstigen Dienstleistungen verknüpften Investitionen mitberücksichtigt, nicht allein im Bank- und Versicherungswesen (11). Sie vermag überdies deutlich zu machen, daß Probleme der Organisation wie der Erbringung von Dienstleistungen in der "Dritten Welt" weithin den in Industriestaaten aufgeworfenen gleichen (s. als Beispiel Telekommunikation: 37, 57 ff., 182 f.). Die Rolle des informellen Sektors wird leider nur bei Zimbabwe kurz gestreift; er läßt sich freilich weniger gut zahlenmäßig erfassen. Dennoch verbindet dieses Buch in gelungener Weise wissenschaftliche Analyse mit Rezepten für die Zukunft und führt überdies dem deutschen Leser vor Augen, wie simpel oft die Entstehung staatlicher wirtschaftlicher Aktivitäten sein kann - man betrachte nur die gleichförmige Herausbildung von Post(spar)banken (62, 123 f., 147, 187, 189), weil nur durch sie flächendeckend Spareinlagen akquiriert werden. 